\title{
Variações dos isótopos de C e Sr em carbonatos pré e pós-glaciação Jequitaí (Esturtiano) na região de Bezerra-Formosa, Goiás
}

\author{
Carlos José Souza de Alvarenga ${ }^{1}$, Maria Emília Schuteski Della Giustina ${ }^{1}$, Nívea Goulart Carramal \\ Silva $^{1}$, Roberto Ventura Santos ${ }^{1}$, Simone Maria Costa Lima Gioia ${ }^{1}$, Edi Mendes Guimarães ${ }^{1}$, Marcel \\ Auguste Dardenne ${ }^{1}$, Alcides Nóbrega Sial ${ }^{2}$ \& Valderez Pinto Ferreira ${ }^{2}$
}

\begin{abstract}
Resumo Na borda ocidental do Cráton do São Francisco são encontradas rochas carbonáticas no topo do Grupo Paranoá e na base do Grupo Bambuí (Formação Sete Lagoas). Essas duas unidades estão separadas pela Formação Jequitaí, entretanto em algumas localidades a ausência do registro glacial dificulta a identificação desses dois carbonatos. Neste trabalho foram selecionadas duas seções onde os carbonatos estão estratigraficamente controlados para as determinações de isótopos de $\mathrm{C}, \mathrm{O}$ e Sr, com o objetivo de se estabelecer valores que possam ser utilizados como ferramenta de correlação. Carbonatos do topo do Grupo Paranoá apresentam valores de $\delta^{13} \mathrm{C}_{\mathrm{PDB}}$ positivos variando entre $+0,8 \mathrm{e}+2,7 \%$, de $\delta^{18} \mathrm{O}_{\mathrm{PDB}}$ variando em sua maior parte entre $-9,0$ e $-4,7 \%$ e e ${ }^{87} \mathrm{Sr} /{ }^{86} \mathrm{Sr}$ com razões entre 0,7063 e 0,7068 . A amostra referente ao diamictito da Formação Jequitaí apresenta valores de $\delta^{13} \mathrm{C}_{\mathrm{PDB}}$ de $-1,8 \%$ e $\delta^{18} \mathrm{O}_{\mathrm{PDB}}-5,1 \%$. Nos carbonatos da Formação Sete Lagoas os dolomitos de capa apresentaram valores de $\delta^{13} \mathrm{C}_{\mathrm{PDB}}$ negativos entre -6,0 e -3,2\%, de $\delta^{18} \mathrm{O}_{\mathrm{PDB}}$ entre $-9,1$ e $-5,3 \%$ e as altas razões de ${ }^{87} \mathrm{Sr} /{ }^{86} \mathrm{Sr}$ sugerindo uma anomalia radiogênica de $\mathrm{Sr}$. Calcários e calcários argilosos acima do dolomito de capa iniciam com valores ainda muito negativos $(-6,0 \%)$ de $\delta^{13} \mathrm{C}_{\mathrm{PDB}}$ passando gradualmente para valores altamente positivos até $+9,2 \%$. Nesse mesmo intervalo os valores de $\delta^{18} \mathrm{O}_{\mathrm{PDB}}$ variam de $-10,1 \%$ na base para $-5,5 \%$ rumo ao topo da formação e as razões de ${ }^{87} \mathrm{Sr} /{ }^{86} \mathrm{Sr}$ mostram dados de boa confiabilidade entre 0,70745 e 0,70758 . Os dados obtidos revelam uma diferença significativa na composição isotópica de carbono entre as rochas carbonáticas do Grupo Paranoá e do Grupo Bambuí, o mesmo acontecendo para as razões de ${ }^{87} \mathrm{Sr} /{ }^{86} \mathrm{Sr}$. Os dados isotópicos aqui obtidos fornecem um modelo de boa resolução para o estabelecimento de novas correlações estratigráficas regionais para as unidades carbonáticas dos grupos Paranoá e Bambuí.
\end{abstract}

Palavras-chave: isótopos de C, isótopos de Sr, dolomitos de capa, Neoproterozóico, glaciação.

\begin{abstract}
C, O$ and Sr Isotopic Stratigraphy of Carbonates Pre and Pos Jequitai Glaciation: Bezerra-Formosa Area, Goiás. Carbonate rocks of the upper Paranoá Group and lower Bambuí Group (Sete Lagoas Formation) have been founded at the occidental border of the São Francisco craton. These units have been separated by the Sturtian glaciation, identified by Jequitaí Formation, but in some localities the glacial record is absent and is difficult to separate these two carbonates units. In this paper two sections with a good stratigraphic control have been chosen to find $\mathrm{C}, \mathrm{O}$ and $\mathrm{Sr}$ isotopic values to have tools for the correlations. Carbonates of the Paranoá Group present a trend of positive $\delta^{13} \mathrm{C}_{\mathrm{PDB}}$ varying between $+0.8 \mathrm{e}+2.7 \%$, of $\delta^{18} \mathrm{O}_{\mathrm{PDB}}$ varying between $-9.0 \mathrm{e}-4.7 \%$ and ${ }^{87} \mathrm{Sr} /{ }^{86} \mathrm{Sr}$ ranging from 0.7063 to 0.7068 . The diamictite sample of Jequitaí Formation has $\delta^{13} \mathrm{C}_{\mathrm{PDB}}$ values of $-1.8 \%$ and $\delta^{18} \mathrm{O}_{\mathrm{PDB}}$ values of $-5.1 \%$. The cap dolomites of the Sete Lagoas Formation are associated with an extremely negative $\delta^{13} \mathrm{C}_{\mathrm{PDB}}$ values between $-6.0 \mathrm{e}-3.2 \%$, $\delta^{18} \mathrm{O}_{\mathrm{PDB}}$ values between -9.1 e $-5.3 \%$ and high ${ }^{87} \mathrm{Sr} r{ }^{86} \mathrm{Sr}$ ratios suggesting a radiogenic $\mathrm{Sr}$ isotope anomaly. Limestone and muddy-limestone above the cap dolomite still starts with extremely negative $\delta^{13} \mathrm{C}_{\mathrm{PDB}}$ values $(-6.0 \%)$ and are followed by an upward trend of increasing carbon isotope values up to $+9.2 \%$. In the same interval the $\delta^{18} \mathrm{O}_{\mathrm{PDB}}$ values ranging from $-10.1 \%$ at the base to $-5.5 \%$ in direction to the top of formation and primary ${ }^{87} \mathrm{Sr}{ }^{86} \mathrm{Sr}$ ratios between 0.70745 and 0.70758 . The Carbon and $\mathrm{Sr}$ isotope data presented here reveal significant differences between carbonates from the Paranoá Group and the Bambuí Group. These data developed here provides the framework for a new regional isotopic correlation model to the carbonates units of the Paranoá and Bambuí groups.
\end{abstract}

Keywords: carbon isotope, $\mathrm{Sr}$ isotope, cap dolomite, Neoproterozoic, glaciation.

INTRODUÇÃO Depósitos de carbonatos tropicais de águas quentes recobrindo rochas glaciogênicas é um paradoxo para a geologia do fim do Neoproterozóico, pois não são comuns mudanças climáticas tão extremas em um curto intervalo de tempo geológico (Hoffman \& Schrag 2002). Atualmente a hipótese da terra totalmente coberta por gelo (snowball Earth) é uma das mais aceita para justificar esse contexto, uma vez que estudos pale- 
omagnéticos confirmam que essas glaciações ocorreram naquela época em baixas latitudes (Hoffman et al. 1998, Hoffman \& Schrag 2002, Hoffman et al. 2002).

Os diamictitos neoproterozóicos, com distribuição em vários continentes, são recobertos por carbonatos de capa, que são geralmente dolomitos. Essas rochas ocorrem em espessuras não superiores a 30 metros, podendo apresentar estromatólitos, brechas e uma estrutura enigmática descrita como giant extreme ripples (Allen \& Hoffman 2005) que também foram descritas como estruturas tepee-like (James et al. 2001, Nogueira et al. 2003). O estudo desses carbonatos de capa é de vital importância para o entendimento das mudanças climáticas no Neoproterozóico (Hoffman \& Schrag 2002).

No centro-leste do Brasil, sobre o Cráton do São Francisco e suas faixas marginais, ocorrem rochas siliciclásticas e carbonáticas mesoproterozóicas do Grupo Paranoá e do Supergrupo Espinhaço sobre as quais são encontrados depósitos glaciais neoproterozóicos, dominados pelos diamictitos nas formações Jequitaí e Bebedouro e por diamictitos que gradam para arenitos e ritmitos no Grupo Macaúbas (Uhlein et al. 1999, 2004, Cukrov et al. 2005). Esses depósitos glaciais estão freqüentemente recobertos por carbonatos pertencentes aos grupos Bambuí e Una. Um empilhamento vertical completo para essas unidades mostra que as unidades carbonáticas mesoproterozóicas e neoproterozóicas estão geralmente separadas pelos depósitos atribuídos à Glaciação Jequitaí, entretanto em várias localidades a ausência do registro glacial tem dificultado a distinção entre os carbonatos dos grupos Paranoá e Bambuí.

Neste trabalho serão apresentadas as determinações petrográficas, análises químicas e isotópicas $(\mathrm{C}, \mathrm{O}, \mathrm{Sr})$ de duas seções estratigráficas de carbonatos estratigraficamente abaixo e acima dos diamictitos glaciais a norte do Povoado de Bezerra (Fig. 1), com o objetivo de propor uma estratigrafia isotópica que permita o estabelecimento de padrões isotópicos distintos para os carbonatos pré e pós Glaciação Jequitaí e assim estabelecer ferramentas que possam contribuir para a distinção de carbonatos de forma a facilitar os estudos de correlação entre seções de uma mesma bacia como entre diferentes bacias sedimentares neoproterozóicas.

CONTEXTO GEOLÓGICO Os grupos Bambuí, Paranoá e a Formação Jequitaí estendem-se por toda borda ocidental do Cráton do São Francisco, ocorrendo tanto no domínio cratônico como na Faixa Brasília. As rochas na faixa dobrada foram deformadas durante a Orogênese Brasiliana, finalizada há aproximadamente 600 Ma (Pimentel et al. 1991). A Formação Jequitaí recobre em discordância as rochas metassedimentares do Grupo Paranoá ou unidades paleoproterozóicas da Formação Ticunzal, Grupo Araí e rochas graníticas do Cráton do São Francisco (Uhlein et al. 2004). Acima dos depósitos glaciogênicos tem-se uma sucessão pelito-carbonatada da Formação Sete Lagoas (FSL) da base do Grupo Bambuí.

O Grupo Paranoá é representado por quartzi- tos, pelitos e carbonatos que foram subdivididos em 11 unidades estratigráficas informais para a região entre São Gabriel e Alto Paraíso (Faria \& Dardenne 1995). A base desse Grupo foi definida por conglomerados com clastos variados suportados por matriz areno-carbonática denominada de Unidade Paraconglomerado São Miguel. Esses conglomerados são recobertos por uma espessa sucessão psamo-pelítica, na qual se intercalam unidades rítmicas (Metarritmitos R1 e R2), compostas por quartzitos, metassiltitos e metargilitos, e unidades quartzíticas (Quartzitos Q1 e Q2) finas a microconglomeráticas bem selecionadas. Acima do Q2 tem-se uma unidade de metassiltitos argilosos laminados, com lentes de quartzito e calcário/dolomito no seu topo. Nas lentes de dolomitos, podem ocorrer estromatólitos do tipo Conophyton. Estudos dos estromatólitos nesse Grupo apresentam uma idade deposicional estimada entre 1170 e $950 \mathrm{Ma}$ (Fairchild et al. 1996).

$\mathrm{Na}$ região de Formosa-Bezerra-Cabeceiras (Fig.1), o Grupo Paranoá é exposto no centro de grandes anticlinais inversos de flancos falhados, podendo estar recoberto pelos diamictitos glaciais da Formação Jequitaí ou diretamente pelos carbonatos do Grupo Bambuí. A Unidade basal do Grupo Paranoá nessa região é composta por quartzitos finos a grossos com selecionamento variável. Por meio de um contato intercalado essa unidade passa ao Ritmito Inferior, que é caracterizado pela alternância de quartzitos finos e metassiltito com freqüentes lentes de calcário e dolomito no seu topo. Um intervalo de arcóseo médio a muito grosso recobre o Ritmito Inferior, sendo recoberto pelo Ritmito Superior formado por alternância de camadas de quartzito, siltito e argilito com ocasionais ocorrências de glauconita (Guimarães et al. 1986).

A Formação Jequitaí na borda ocidental do Cráton do São Francisco apresenta uma distribuição irregular com locais onde seus depósitos estão ausentes e em outros podendo ultrapassar os $150 \mathrm{~m}$ de espessura (Uhlein et al. 2004, Cukrov et al. 2005). Na região de Bezerra-Cabeceiras suas rochas incluem diamictitos e arenitos líticos, bem como siltitos e argilitos vermelhos que podem conter pequenos fragmentos de rocha (Guimarães 1997). O diamictito, principal rocha dessa formação, é composto por clastos angulosos de dimensões variáveis de quartzito, arcóseo, chert e dolomito, dispersos em uma matriz síltico-argilosa (Guimarães et al. 1986), e sua origem é interpretada como decorrente de fluxos gravitacionais em ambiente glácio-marinho (Uhlein et al. 2004). A fase continental dessa glaciação está representada por pavimentos estriados, encontrados na região da cidade de Jequitaí, que indicam o movimento da geleira de oeste para leste (Isotta et al. 1969, Karfunkel et al. 2003, Cukrov et al. 2005).

O Grupo Bambuí, definido por Branco \& Costa (1961) foi individualizado em três megaciclos regressivos shallowing-upward, cada um representado por um período de transgressão, seguido de subsidência da bacia e por fim uma regressão marinha (Dardenne 1978, 1981, 2000). O primeiro megaciclo inclui a Formação Sete Lagoas, que consiste de calcários, dolomi- 


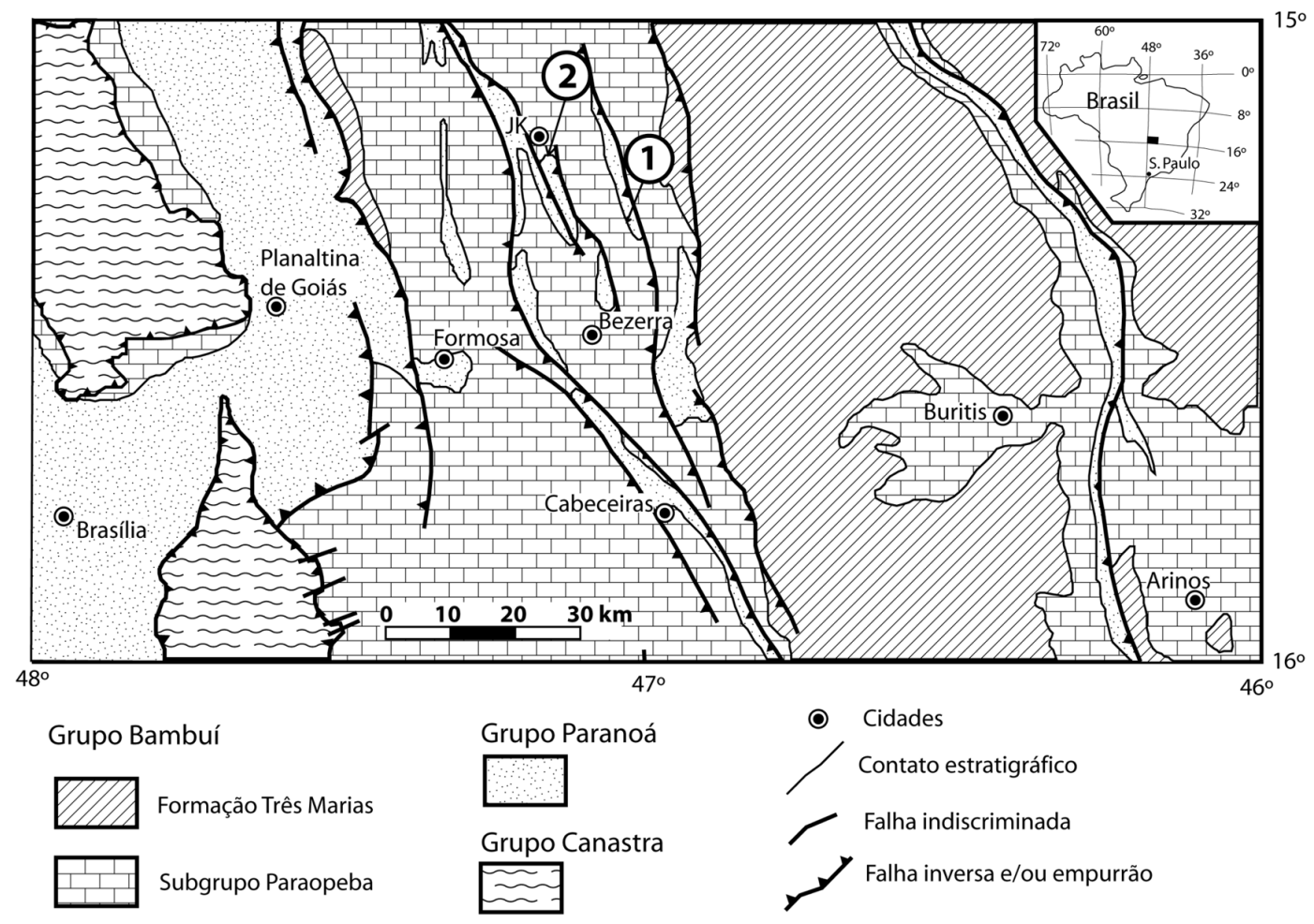

Figura 1 - Mapa geológico mostrando a distribuição das rochas do Neoproterozóico da região entre Brasília, Formosa e Buritis. 1: Localização da seção Fazenda Limoeiro. 2: Localização da seção do Povoado JK. (Adaptado de Souza et al. 2004).

tos, margas e siltitos calcíferos que em vários locais recobre diretamente os diamictitos glaciais. Os arenitos finos e ritmitos pelíticos da Formação Serra de Santa Helena, seguidos pelos calcários e margas da Formação Lagoa do Jacaré, constituem o segundo megaciclo. O último megaciclo caracteriza-se por argilitos da Formação Serra da Saudade que passam gradualmente para os siltitos arcoseanos e arcóseos finos da Formação Três Marias. A denominação Subgrupo Paraopeba é dada para o conjunto das rochas pelito carbonatadas do Grupo Bambuí. Datação $\mathrm{Pb} / \mathrm{Pb}$ em carbonato indeformado da Formação Sete Lagoas evidencia uma idade de 740 $\pm 22 \mathrm{Ma}$ (Babinski et al. 2007), dado que sugere que os diamictitos subjacentes sejam relacionados à Glaciação Esturtiana ( $730 \mathrm{Ma}$.).

A separação do supercontinente Rodínia no final do Mesoproterozóico resultou uma bacia do tipo riftemargem passiva para a margem oeste do Cráton do São Francisco, na qual foram depositadas as rochas sedimentares e químicas que compõem o Grupo Paranoá (Valeriano et al. 2004). As mudanças climáticas ocorridas no Neoproterozóico (Criogeniano) estabeleceram um regime de depósitos glaciogênicos na Formação Jequitaí. A resposta flexural aos processos deformacionais iniciados na Faixa Brasília deram origem a uma bacia do tipo foreland, na qual se depositaram os sedimentos que compõem o Grupo Bambuí (Guimarães \& Dardenne 2004, 2005). A presença de plagioclásios nos siltitos e quartzitos muito finos da Formação Serra de Santa Helena é atribuída a uma fonte sedimentar a oeste, relacionada ao Arco Magmático de Goiás, devido a uma inversão tec- tônica (Guimarães 1997). Leques de conglomerados e arcóseos no extremo sul do Grupo Bambuí são também relacionados à bacia foreland (Castro 1997, Castro \& Dardenne 2000). O aumento abrupto dos valores de $\delta^{13} \mathrm{C}$ no topo da Formação Sete Lagoas foram interpretados como um período de restrição da Bacia Bambuí como conseqüência da inversão tectônica (Santos et al. 2000). A Formação Três Marias é a unidade que representa o preenchimento final da bacia de sedimentação do Grupo Bambuí (Martins-Neto \& Alkmim 2001).

AMOSTRAGEM E MÉTODOS ANALÍTICOS As amostras foram coletadas na região a norte do povoado de Bezerra (Fig.1) em dois perfis que apresentam um dolomito de capa recobrindo diretamente diamictitos. Quinze amostras são do perfil JK e onze estão na seção Limoeiro. Todas as amostras foram submetidas a análises químicas de elementos maiores e de isotópos de C, $\mathrm{O}$ e $\mathrm{Sr}$.

As amostras foram determinadas por perda ao fogo e por fusão com tetraborato de lítio. As leituras foram realizadas em espectrômetro de fluorescência de raios-X Rigaku, modelo RIX 3000, equipado com tubo de $\mathrm{Rh}$, do LABISE-UFPE. Os resultados obtidos foram recalculados para $100 \%$ para considerar a perda ao fogo.

As razões isotópicas de carbono e oxigênio foram obtidas após a reação com $\mathrm{H}_{3} \mathrm{PO}_{4} 100 \%$ a $25^{\circ} \mathrm{C}$ durante no mínimo 12 horas para a calcita e 3 dias para a dolomita. As amostras foram então evaporadas em um tubo de vácuo, no qual o $\mathrm{CO}_{2}$ liberado foi separado. As análises de $\delta^{13} \mathrm{C}$ e $\delta^{18} \mathrm{O}$ da seção JK foram realizadas em um espectrômetro de massa com fonte de gás, 
marca Finnigan Delta E, do Laboratório de Isótopos Estáveis da Universidade de São Paulo. As amostras da seção Limoeiro foram analisadas pelo mesmo método no LABISE-UFPE.

Para a análise de ${ }^{87} \mathrm{Sr} /{ }^{86} \mathrm{Sr} 50 \mathrm{mg}$ de amostra pulverizada foram digeridas por ácido acético $0,5 \mathrm{~N}$. Após segundo ataque ácido com $\mathrm{HCl} 2,5 \mathrm{~N}$ o $\mathrm{Sr}$ foi separado em colunas orgânicas primárias grandes. A razão ${ }^{87} \mathrm{Sr} /{ }^{86} \mathrm{Sr}$ das amostras foi determinada pelo espectrômetro de massa de ionização termal, marca Finnigan MAT 262, no Laboratório de Geocronologia da Universidade de Brasília.

SEÇÕES ESTUDADAS As duas seções estudadas, distantes uma da outra de 20 quilômetros, localizamse uma no Povoado JK e outra na Fazenda Limoeiro (Fig.1) e foram escolhidas por apresentarem afloramentos com dolomitos de capa da Formação Sete Lagoas em contato direto sobre os diamictitos. As rochas do Grupo Paranoá estão sobrepostas pelo diamictito glaciogênico da Formação Jequitaí, que por sua vez é recoberto por uma sucessão carbonática de aproximadamente 250 metros da Formação Sete Lagoas. No topo da seção da Fazenda Limoeiro ocorrem siltitos da Formação Serra de Santa Helena. Essas seções estão localizadas no domínio externo da Faixa Brasília e encontram-se situadas em flancos de dobras (Fig.1).

Grupo Paranoá As rochas carbonáticas do Grupo Paranoá, descritas na seção do Povoado JK têm aproximadamente 28 metros de espessura (Fig.2 B) e estão estratigraficamente posicionadas no topo da unidade de Ritmito Inferior (Guimarães et al. 1986). Os primeiros 4 metros são formados por dololutitos e calcilutitos dolomíticos laminados com ocasionais filmes argilosos intercalados, que são sucedidos por aproximadamente
20 metros de calcilutitos e margas com ocasionais lâminas de calcarenitos. Os 3 metros superiores dos carbonatos incluem calcilutitos capeados por uma camada de 10 centímetros de dololutito calcífero homogêneo de aspecto maciço. Arcóseo médio a grosso com aproximdamente 40 metros de espessura recobre a unidade carbonática, representando o topo desse grupo na seção do Povoado JK.

Formação Jequitaí A Formação Jequitaí apresenta nas duas seções uma espessura variável entre 6 a 15 metros, que sobrepõe por contato brusco, os quartzitos arcoseanos do Grupo Paranoá (Fig.2). O diamictito maciço é a rocha dominante dessa formação que inclui fragmentos angulosos de calcário, dolomito, quartzito e feldspato, variando de tamanho milimétrico até 0,5 $\mathrm{m}$, dispersos em uma matriz siltica-argilosa carbonática (Fig. 3).

Grupo Bambuí O Grupo Bambuí está presente nas duas seções com a Formação Sete Lagoas sobrepondo os diamictitos da Formação Jequitaí. Na seção JK, os afloramentos permitem um bom controle estratigráfico apenas para a base da formação, enquanto que na Fazenda Limoeiro o estudo foi feito em uma seção completa da formação (Fig. 2). O contato basal da Formação Sete Lagoas recobrindo diretamente os diamictitos glaciais é raramente exposto, fato que torna as seções descritas neste trabalho de grande importância para a compreensão do início da sedimentação carbonática do Grupo Bambuí. Os primeiros 7 metros de espessura são formados por um dolomito de capa composto por um dololutito microcristalino muito homogêneos de cor rosada a cinza, que apesar de apresentar um aspecto aparentemente maciço devido a sua homogeneidade composicional, mostram

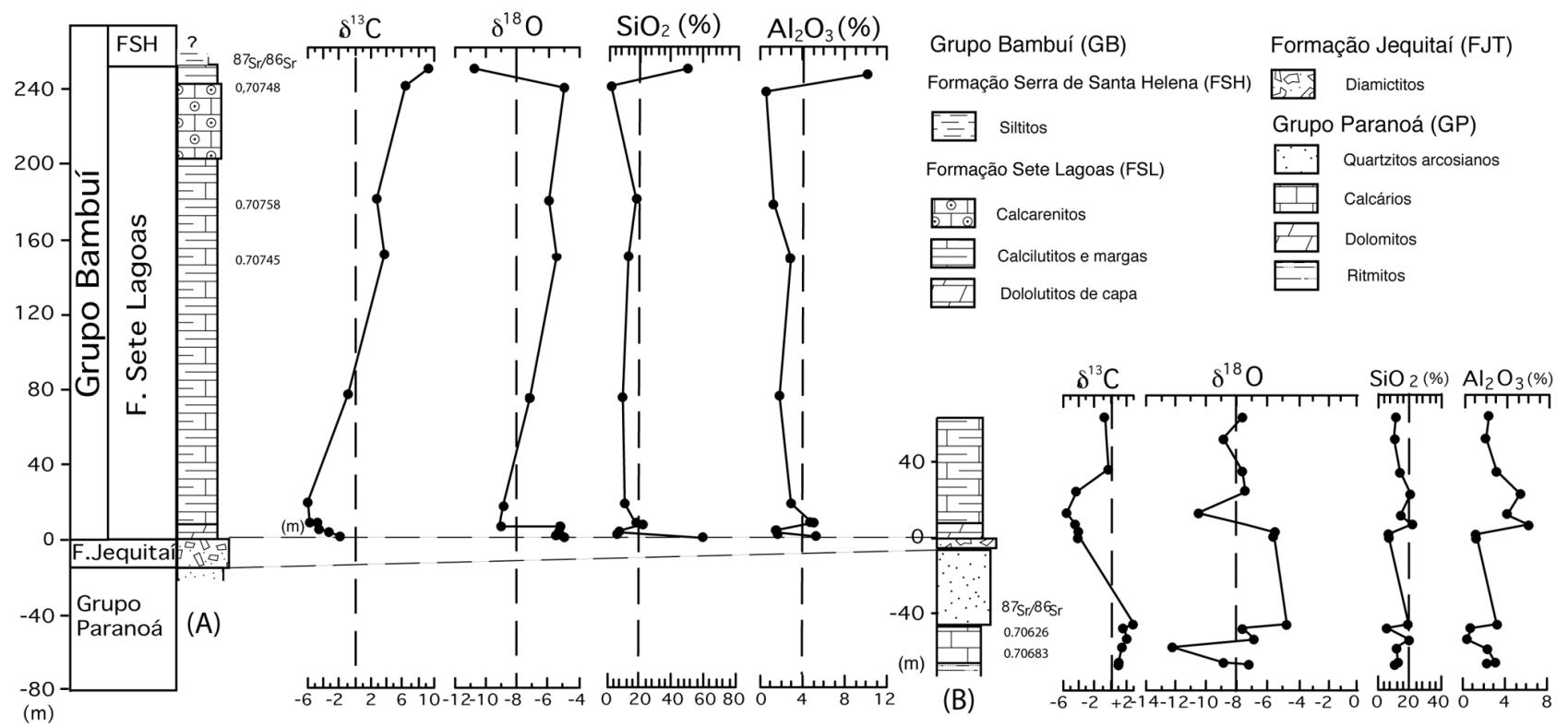

Figura 2 - Seções estratigráficas e variações de $\delta^{13} C_{P D B^{3}} \delta^{18} \mathrm{O}_{P D B^{\circ}}{ }^{87} \mathrm{Sr}{ }^{86} \mathrm{Sr}$ e dados geoquímicos dos carbonatos dos grupos Paranoá e Bambuí. A: Seção Fazenda Limoeiro. B: Seção do Povoado JK. Ver a Fig. 1 para a localização das seções. 
evidências em algumas amostras de uma estrutura laminada.

Acima do dolomito de capa ocorre uma seção de aproximadamente 200 metros de espessura que se inicia por calcilutitos e calcarenitos interclados com lâminas e camadas margosas de cor avermelhada e esverdeada, que mudam para a cor cinza e cinza escuro em direção ao topo (Fig. 4). Laminações cavalgantes e truncamentos por ondas são preservados em algumas camadas de calcarenitos. $\mathrm{Na}$ escala microscópica são comuns os calcários com micropelóides intercalados com lâminas margosas.

Os 40 metros de espessura do topo da Formação Sete Lagoas são formados por calcarenito cinza escuro com lâminas de oolitos e pelóides (Fig. 2 A). Petrograficamente os pelóides são de dois tipos: (1) claros, paralelos à laminação e idênticos aos micropelóides encontrados mais para a base da formação e (2) escuros que ocorrem de forma irregular na rocha, em aglomerados ou dispersos (Fig. 5). Pelóides escuros, semelhantes aos descritos neste trabalho, foram também encontrados em grainstone-wackestone do Neoproterozóico da Formação Hayhook no noroeste do Canadá (James et al. 2001).

A Formação Serra de Santa Helena é caracterizada pelas intercalações de siltitos e siltitos arenosos amarelados quando afetados por intemperismo e acinzentados quando frescos. O contato inferior dessa formação com os calcários da Formação Sete Lagoas é gradual por intermédio de margas e siltitos calcíferos (Fig. 2 A).

RESULTADOS A composição química das amostras (Tab.1) foi usada principalmente para avaliar os efeitos dos processos pós-deposicionais, baseados nas razões de $\mathrm{Mn} / \mathrm{Sr}$ e na quantidade de Sr. Identificar o quanto as rochas foram afetadas por modificações pós-deposicionais é particularmente importante para avaliar se os valores isotópicos primários foram preservados.

Isótopos de carbono e oxigênio Os dolomitos e calcários dolomíticos do Grupo Paranoá possuem valores de $\delta^{13} \mathrm{C}_{\mathrm{PDB}}$ entre $+0,74 \mathrm{e}+2,67 \%$, com aumento gradativo rumo ao topo (Tab.1). Os valores de $\delta^{18} \mathrm{O}_{\mathrm{PDB}}$ apresentam um decréscimo inicial de -7,26\% a -12,32\%o, seguido por uma elevação até o valor de $-4,74 \%$ nos calcários próximos ao contato superior com os quartzitos arcosianos (Fig. 2, Tab.1).

Valor de $\delta^{13} \mathrm{C}_{\mathrm{PDB}}$ negativo foi obtido da matriz de uma amostra de diamictito maciço da Formação Jequitaí. Esse valor deve estar refletindo a mistura da composição isotópica dos clastos carbonáticos e do cimento, porque os fragmentos de calcários menores que $2 \mathrm{~mm}$ e o cimento carbonático da matriz foram amostrados juntos.

O dolomito de capa da base da Formação Sete Lagoas apresenta valores de $\delta^{13} \mathrm{C}_{\mathrm{PDB}}$ entre -3\% a -5\%, mostrando uma queda gradual para valores mais negativos no seu topo (Fig. 2). Nos calcários e margas, que sucedem estratigraficamente o dolomito de capa, ainda persistem valores negativos de $\delta^{13} \mathrm{C}_{\mathrm{PDB}}$ por aproximadamente 68 metros, porém já indicando um aumento

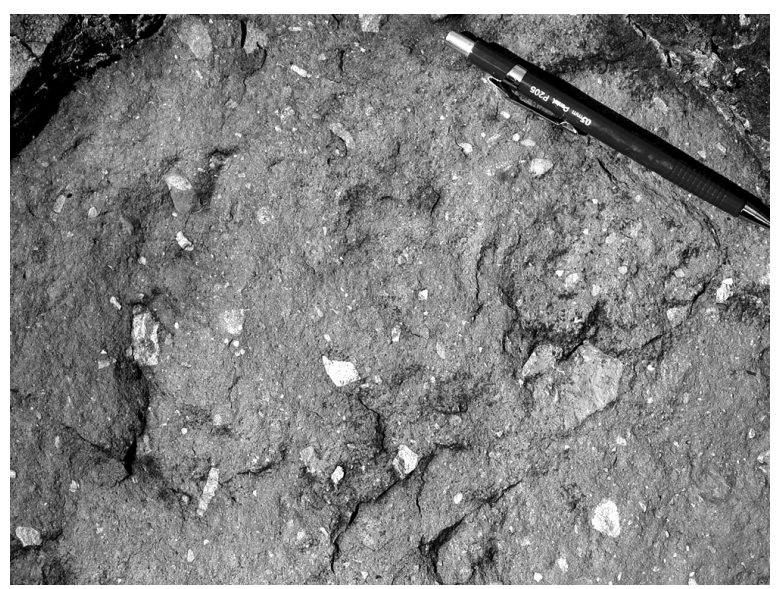

Figura 3 - Diamictito glacial da Formação Jequitai. Observar o formato anguloso dos fragmentos dispersos em uma matriz síltico-argilosa carbonática.

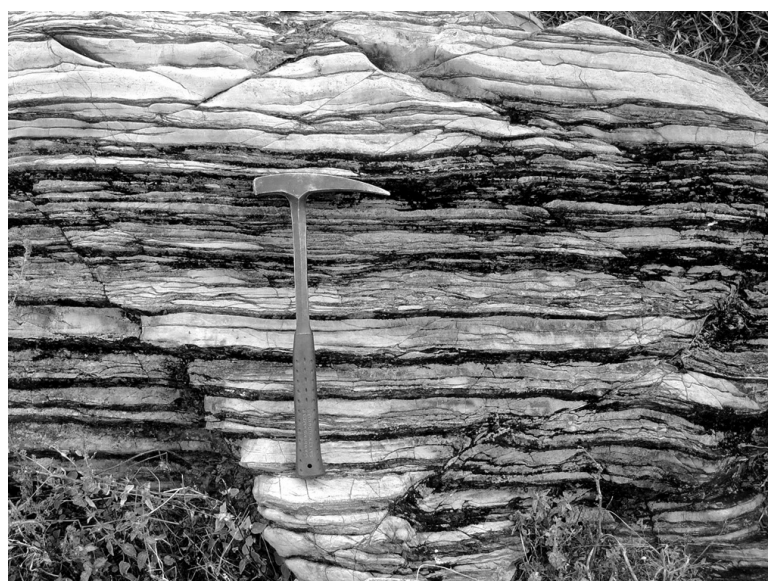

Figura 4 - Calcilutitos laminados da Formação Sete Lagoas, Grupo Bambuí. As lâminas escuras são de margas intercalados em calcários. Localizada 20 metros acima dos diamictitos na seção da Fazenda Limoeiro.

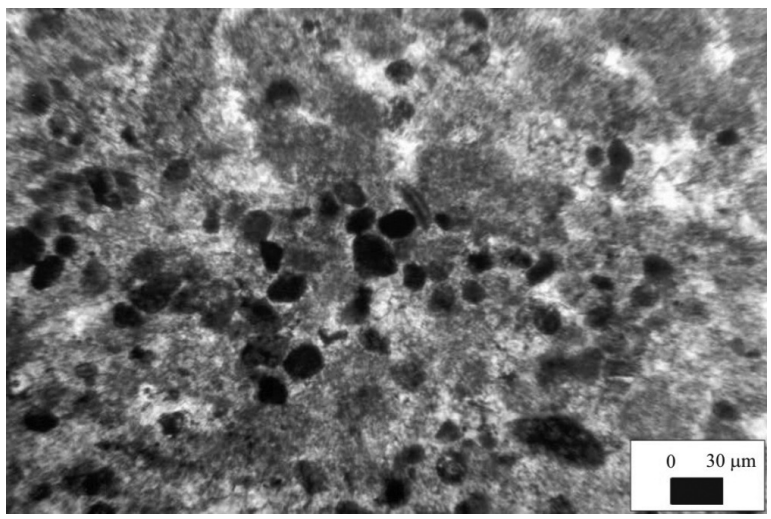

Figura 5 - Calcarenitos do topo da Formação Sete Lagoas, Grupo Bambuí. Pelóides claros micríticos e pelóides escuros em aglomerados (porção central da foto) e dispersos. 
Tabela 1 - Isótopos de carbono, oxigênio, Sr e composição química de amostras das seções da Fazenda Limoeiro e do Povoado JK.

\begin{tabular}{|c|c|c|c|c|c|c|c|c|c|c|c|c|c|}
\hline Seção & $\begin{array}{c}\text { Unidade } \\
\text { Estratigrá- } \\
\quad \text { fica }\end{array}$ & Amostra & $\begin{array}{l}\text { Lito- } \\
\text { logia }\end{array}$ & $\begin{array}{l}\text { Espes- } \\
\text { sura } \\
(\mathrm{m})\end{array}$ & $\begin{array}{c}\mathrm{SiO}_{2} \\
(\%)\end{array}$ & $\begin{array}{c}\mathrm{Al}_{2} \mathrm{O}_{3} \\
(\%)\end{array}$ & $\begin{array}{c}\stackrel{d}{ }^{13} C \\
(P D B)\end{array}$ & $\begin{array}{c}d^{18} \mathrm{O} \\
(P D B)\end{array}$ & $\begin{array}{c}\mathrm{Mg} / \\
\mathrm{Ca}\end{array}$ & $\begin{array}{c}\text { Mn } \\
\text { (ppm) }\end{array}$ & $\begin{array}{c}S r \\
(p p m)\end{array}$ & $\begin{array}{c}M n / \\
S r\end{array}$ & ${ }^{87} \mathrm{Sr}{ }^{\beta 6} \mathrm{Sr}$ \\
\hline \multirow{6}{*}{$\begin{array}{l}\mathbf{L} \\
\mathbf{I} \\
\mathbf{M} \\
\mathbf{O}\end{array}$} & \multirow{10}{*}{$\begin{array}{c}\text { Grupo } \\
\text { Bambuí }\end{array}$} & Bz 39 & Calci. & 250,0 & 50,0 & 10,4 & 9,2 & $-10,8$ & 0,23 & 1495 & 942 & 1,59 & 0,70797 \\
\hline & & $\mathrm{Bz} 38$ & Calcare. & 240,0 & 2,7 & 0,7 & 6,3 & $-5,0$ & 0,04 & 39 & 1990 & 0,02 & 0,70748 \\
\hline & & Bz 37 & Calci. & 180,0 & 18,1 & 1,3 & 2,7 & $-6,0$ & 0,09 & 215 & 1616 & 0,13 & 0,70758 \\
\hline & & $\mathrm{Bz} 36$ & Calci. & 150,0 & 13,9 & 3,0 & 3,6 & $-5,5$ & 0,04 & 260 & 3064 & 0,08 & 0,70745 \\
\hline & & $\mathrm{Bz} 35$ & Calci. & 75,0 & 10,6 & 1,8 & $-0,9$ & $-7,3$ & 0,05 & 151 & 191 & 0,79 & 0,70808 \\
\hline & & $\mathrm{Bz} 18 \mathrm{~F}$ & Calci. & 17,0 & 11,3 & 2,9 & $-6,0$ & $-8,9$ & 0,03 & 177 & 211 & 0,84 & 0,70855 \\
\hline \multirow{5}{*}{$\begin{array}{l}\mathbf{E} \\
\mathbf{I} \\
\mathbf{R} \\
\mathbf{O}\end{array}$} & & Bz 18E & Calci. & 7,0 & 18,5 & 5,1 & $-5,7$ & $-9,1$ & 0,17 & 307 & 207 & 1,48 & 0,71097 \\
\hline & & $\mathrm{Bz} 18 \mathrm{D}$ & Dolol. & 6,5 & 21,8 & 5,1 & $-4,7$ & $-5,3$ & 0,68 & 454 & 115 & 3,95 & ND \\
\hline & & $\mathrm{Bz} 18 \mathrm{C}$ & Dolol. & 3,0 & 9,5 & 1,6 & $-4,4$ & $-5,4$ & 0,71 & 244 & 95 & 2,57 & ND \\
\hline & & $\mathrm{Bz} 18 \mathrm{~B}$ & Dolol. & 1,0 & 6,8 & 1,4 & $-3,2$ & $-5,6$ & 0,64 & 209 & 164 & 1,27 & 0,71058 \\
\hline & $\begin{array}{c}\text { Formação } \\
\text { Jequitaí }\end{array}$ & Bz 18A & Diamic. & $-1,0$ & 60,0 & 5,0 & $-1,8$ & $-5,1$ & 0,83 & 652 & 42 & 15,52 & ND \\
\hline \multirow{15}{*}{ K } & \multirow{8}{*}{$\begin{array}{c}\text { Grupo } \\
\text { Bambuí }\end{array}$} & JK 17 & Calci. & 64,0 & 12,0 & 2,5 & $-0,9$ & $-7,8$ & 0,03 & 245 & 145 & 1,69 & ND \\
\hline & & JK 16 & Calci. & 52,0 & 10,7 & 2,3 & $-0,7$ & $-9,0$ & 0,04 & 193 & 148 & 1,30 & ND \\
\hline & & JK 15 & Calci. & 40,0 & 14,8 & 3,2 & $-0,4$ & $-7,8$ & 0,12 & 382 & 250 & 1,53 & 0,70932 \\
\hline & & JK 14 & Calci. & 28,0 & 22,1 & 5,4 & $-4,4$ & $-7,6$ & 0,29 & 780 & 195 & 4,00 & ND \\
\hline & & JK 13 & Calc. & 16,0 & 15,5 & 4,2 & $-5,7$ & $-10,6$ & 0,05 & 265 & 141 & 1,88 & ND \\
\hline & & JK 12 & Dolol. & 7,0 & 22,6 & 6,1 & $-4,6$ & $-7,6$ & 0,56 & 348 & 100 & 3,48 & ND \\
\hline & & JK 11 & Dolol. & 3,0 & 7,3 & 1,5 & 4,3 & $-5,7$ & 0,58 & 300 & 58 & 5,17 & ND \\
\hline & & JK 10 & Dolol. & 0,0 & 7,3 & 1,5 & $-4,2$ & $-5,6$ & 0,58 & 241 & 64 & 3,77 & ND \\
\hline & \multirow{7}{*}{$\begin{array}{c}\text { Grupo } \\
\text { Paranoá }\end{array}$} & JK 9 & Calc. & $-46,0$ & 20,8 & 3,3 & 2,7 & $-4,7$ & 0,47 & 379 & 148 & 2,56 & ND \\
\hline & & JK 8A & Calc. & $-49,0$ & 21,5 & 4,2 & 1,4 & $-7,7$ & 0,04 & 172 & 232 & 0,74 & ND \\
\hline & & JK 8 & Calc. & $-49,0$ & 6,1 & 1,0 & 1,5 & $-7,6$ & 0,02 & 196 & 472 & 0,42 & 0,70626 \\
\hline & & JK 7 & Calc. & $-55,0$ & 21,3 & 0,5 & 1,9 & $-6,9$ & 0,14 & 121 & 479 & 0,25 & 0,70683 \\
\hline & & JK 6 & Calc. & $-60,0$ & 12,6 & 2,4 & 1,2 & $-12,3$ & 0,05 & 170 & 90 & 1,89 & ND \\
\hline & & JK 3B & Dolol. & $-70,0$ & 13,6 & 3,1 & 0,7 & $-9,0$ & 0,22 & 350 & 81 & 4,32 & ND \\
\hline & & JK 1 & Dolol. & $-74,0$ & 12,1 & 2,3 & 0,8 & $-7,3$ & 0,52 & 802 & 63 & 12,73 & ND \\
\hline
\end{tabular}

Litologias: Calc. - Calcário, Calci. - Calcilutito, Calcare. - Calcarenito, Diamic. - Diamictito, Dolol. - Dololutito

da razão isotópica para $-0,4 \%$ o (Fig. 2). Em direção ao topo da Formação Sete Lagoas, os calcários tornam-se cinza escuro a preto, com um maior teor de matéria orgânica que os calcários precedentes e apresentam valores de $\delta^{13} \mathrm{C}_{\mathrm{PDB}}$ entre $+2,7$ e $+9,0 \%$. Os valores de $\delta^{18} \mathrm{O}_{\mathrm{PDB}}$ dos dolomitos de capa são próximos à $-5,5 \% \mathrm{e}$ um rápido decréscimo desse valor marca o início dos calcários, com valores de $\delta^{18} \mathrm{O}_{\mathrm{PDB}}$ entre $-8,9$ e $-10,1 \%$ gradacionando para $-5,5 \%$ rumo ao topo da seção. A queda brusca do valor de $\delta^{18} \mathrm{O}_{\mathrm{PDB}}$ para $-10,8 \%$ foi observada na amostra do topo da seção (Fig. 2 A) que contém proporção elevada de terrígenos (Tab.1).

Isótopos de estrôncio Os calcários do topo do Grupo Paranoá forneceram razões de ${ }^{87} \mathrm{Sr} /{ }^{86} \mathrm{Sr}$ entre 0,70683 e 0,70626 , enquanto que as razões isotópicas para os carbonatos do Grupo Bambuí variam entre 0,70745 e 0,71058. Os dolomitos de capa da FSL fornecem altas razões de ${ }^{87} \mathrm{Sr} /{ }^{86} \mathrm{Sr}(\sim 0,71058)$, baixo conteúdo de $\mathrm{Sr}$ $(<164 \mathrm{ppm})$ e altas razões $\mathrm{Mn} / \mathrm{Sr}$ superior a 1,3 (Tab.1). Os 75 metros de calcários e margas acima do dolomito de capa apresentam razão ${ }^{87} \mathrm{Sr} /{ }^{86} \mathrm{Sr}$ entre $0,70932 \mathrm{e}$ 0,70808 , baixos conteúdos de $\mathrm{Sr}(<211 \mathrm{ppm})$ e razões de $\mathrm{Mn} / \mathrm{Sr}$ superiores a 0,8 (Tab.1). Calcário com alto teor de $\mathrm{Sr}(1616-3064 \mathrm{ppm})$ e razões de $\mathrm{Mn} / \mathrm{Sr}$ entre 0,02 e 0,13 forneceram razões ${ }^{87} \mathrm{Sr} /{ }^{86} \mathrm{Sr}$ entre 0,70745 e 0,70758 .

DISCUSSÕES O Mesoproterozóico e o Neoproterozóico são marcados por diferenças nos valores de $\delta^{13} \mathrm{C}_{\mathrm{PDB}}$ de seus carbonatos marinhos (Veizer et al. 1980, Kha et al. 1999). Enquanto carbonatos mesoproterozóicos são conhecidos por ter valores de isótopos de carbono próximos de $0 \%, \delta^{13} \mathrm{C}_{\mathrm{PDB}}$ nas rochas neoproterozóicas tem valores variando de -12 a $+13 \%$ ou maiores (Knoll et al. 1986, Margaritz et al. 1986, Kaufman \& Knoll 1995, Iyer et al. 1995, Buick et al. 1995, Santos et al. 2000, 2004). Os baixos valores de $\delta^{13} C_{\mathrm{PDB}}$ estão associados com os eventos glaciais, enquanto que altos valores estão geralmente relacionados com grande quantidade de deposição de matéria orgânica enriquecendo o reservatório de carbono residual (Knoll et al. 1986).

Os dados isotópicos apresentados neste trabalho revelam diferenças significativas entre a composição isotópica das rochas mesoproterozóicas do Grupo Paranoá e as rochas neoproterozóicas do Grupo Bambuí (Fig. 2, Tab.1). Os valores isotópicos de carbono para os carbonatos do Grupo Paranoá apresentam apenas 
dados positivos, ligeiramente crescentes para o topo e restritos a um intervalo entre $+0,7$ e $+2,7 \%$. Os isótopos de Carbono encontrados para o Grupo Paranoá, neste trabalho estão de acordo com valores obtidos para os carbonatos mesoproterozóicos dos grupos Paranoá e Espinhaço, reforçando a proposta de que valores de $\delta^{13} \mathrm{C}_{\mathrm{PDB}}$ variando entre $0,0 \mathrm{e}+2 \%$ o são representativos para essas duas unidades mesoproterozóicas (Santos et al. 2000, Santos et al. 2004).

$\mathrm{O}$ comportamento da razão $\delta^{13} \mathrm{C}_{\mathrm{PDB}}$ na base do Grupo Bambuí, inicialmente negativo nos dolomitos de capa é seguido por uma forte incursão positiva rumo aos calcários do topo, apontando para diferenças nas condições paleoambientais durante a deposição. Os valores negativos de $\delta^{13} \mathrm{C}_{\mathrm{PDB}}$ nos primeiros 7 metros que recobrem os diamictitos são compatíveis com o intervalo do dolomito de capa (cap dolomite). Estruturas sedimentares, tais como estromatólitos, tubos de gás, giante wave ripples e leques de Aragonita, têm sido recentemente descritas para os dolomitos de capa que recobrem os depósitos da Glaciação Marinoana de idade em torno de $635 \mathrm{Ma}$ (Nogueira et al. 2003, Alvarenga et al. 2004, Allen \& Hoffman 2005, Alvarenga et al. 2007), entretanto para os carbonatos de capa relacionados à Glaciação Esturtiana em torno de $730 \mathrm{Ma}$, tais estruturas não são conhecidas (Gorjan et al. 2003, Hoffman \& Schag 2002, Yoshioka et al. 2003, Halverson et al. 2005), reforçando assim a provável idade Esturtiana para a Glaciação Jequitaí. Os calcários que seguem os dolomitos de capa possuem valores de $\delta^{13} \mathrm{C}_{\mathrm{PDB}}$ mais elevados, o que sugere o início de um clima quente imediatamente após o término do período glacial. Os valores da razão isotópica de carbono permitem interpretar um cenário pós-glacial submetido a um aquecimento e aumento da matéria orgânica no sistema marinho. Esse comportamento de aumento gradual do $\delta^{13} \mathrm{C}_{\mathrm{PDB}}$ é comum nas seções pós-esturtianas do Subgrupo Abenab na Namíbia, Grupo Umberatana na Austrália e nas formações Twitya e Keele no Canadá (Hill \& Walter 2000, James et al. 2001, McKirdy et al. 2001, Hoffman \& Schrag 2002, Halverson et al. 2005).

Os dados de ${ }^{87} \mathrm{Sr} /{ }^{86} \mathrm{Sr}$ geralmente mostram variações com a composição da rocha devendo serem usados com cuidado na interpretação primária da sedimentação (Hoffman et al. 1998, Fairchild et al. 2000, James et al. 2001, Alvarenga et al. 2004, 2007). Neste trabalho os dolomitos apresentaram baixo conteúdo de $\mathrm{Sr}$, altas razões de $\mathrm{Mn} / \mathrm{Sr}$ e altas razões de ${ }^{87} \mathrm{Sr} /{ }^{86} \mathrm{Sr}$ que têm sido interpretado como devido a efeitos pós-deposicionais. Os calcários do Grupo Paranoá e da Formação Sete Lagoas apresentaram um amplo intervalo de razões de ${ }^{87} \mathrm{Sr} /{ }^{86} \mathrm{Sr}(0,70626-0,71097)$, e os valores primários foram considerando de acordo com a quantidade de Sr e a razão $\mathrm{Mn} / \mathrm{Sr}$ na rocha. Nas duas amostras de calcário do Grupo Paranoá o conteúdo de $\mathrm{Sr}$ foi superior a $470 \mathrm{ppm}$, as razões $\mathrm{Mn} / \mathrm{Sr}$ inferiores a 0,45 e as razões ${ }^{87} \mathrm{Sr}{ }^{86} \mathrm{Sr}$ de valores próximos $(0,70626-0,70683)$, sugerindo se tratar de dados primários. As razões ${ }^{87} \mathrm{Sr} /{ }^{86} \mathrm{Sr}$ encontradas para os carbonatos do Grupo Paranoá, quando comparadas a outras seções mundiais, podem sugerir uma idade deposicional entre 650-900 Ma (Gorokhov et al. 1995, Jacobsen \& Kaufman 1999, Walter et al. 2000, Thomas et al. 2004, Halverson et al. 2005), mas calcários de idade mesoproterozóica com razões de ${ }^{87} \mathrm{Sr} /{ }^{86} \mathrm{Sr}$ dessa ordem tem também sido encontrados na Sibéria (Bartley et al. 2001). Diante de tais dados os valores de $\mathrm{Sr}$ não podem ser usados como ferramenta isolada na determinação de idades, devendo sua interpretação considerar os isótopos de $\mathrm{C}$ e o compotamento estratigráfico bacinal.

Nas amostras de calcário da Formação Sete Lagoas que apresentam elevados valores de $\mathrm{Mn} / \mathrm{Sr}$ superior a 0,80 , baixo conteúdo de $\mathrm{Sr}$ inferior a $250 \mathrm{ppm}$ e o alto conteúdo de terrígenos superior a $60 \%$ foram interpretadas como resultado da interferência de processos de alteração pós deposicionais. Entretanto, três amostras da parte superior da seção da Formação Sete Lagoas mostraram razões de ${ }^{87} \mathrm{Sr} /{ }^{86} \mathrm{Sr}$ muito próximas (0,70745-0,70758) que são acompanhadas de critérios geoquímicos altamente favoráveis para uma interpretação de que essas razões refletem condições primárias (Tab.1). Dados isotópicos de estrôncio para as rochas neoproterozóicas dos grupos Bambuí e Una são sempre superiores a 0,70740 (Misi \& Veizer 1998, Misi 2001), e compatíveis com os valores obtidos neste trabalho (Fig. 2).

CONCLUSÃO Isótopos de Carbono e Estrôncio mostraram que as significativas diferenças entre esses valores estão relacionadas com a estratigrafia. Enquanto os valores de $\delta^{13} \mathrm{C}_{\mathrm{PDB}}$ são sempre positivos e não variam mais que 3,0\% para os carbonatos do Grupo Paranoá, os valores para os carbonatos da Formação Sete Lagoas flutuam em até $15,0 \%$. As duas seções estudadas mostraram correspondência nos valores de $\delta^{13} \mathrm{C}_{\mathrm{PDB}}$ para a Formação Sete Lagoas, mostrando em ambas uma diminuição gradual do $\delta^{13} \mathrm{C}_{\mathrm{PDB}}$ para o topo do carbonato de capa, seguidos por um aumento gradual dos valores nos carbonatos acima da capa até atingir seu valor máximo no topo da formação.

As razões de ${ }^{87} \mathrm{Sr} /{ }^{86} \mathrm{Sr}$ para os calcários do Grupo Paranoá mostram valores distintos daqueles encontrados para as rochas do Grupo Bambuí. As razões de ${ }^{87} \mathrm{Sr} /{ }^{86} \mathrm{Sr}$ para os carbonatos do Grupo Bambuí estão de acordo com os valores já conhecidos fora da área de estudo relacionados uma plataforma carbonática neoproterozóica, instalada logo após a glaciação esturtiana ( $\sim 750 \mathrm{Ma}$.).

Os dados isotópicos deste trabalho revelam que o uso da investigação de isótopos de $\mathrm{C}$ e $\mathrm{Sr}$ pode auxiliar na distinção entre carbonatos dos grupos Paranoá e Bambuí quando a relação estratigráfica deles com os diamictitos da Formação Jequitaí não for identificada.

Agradecimentos Os autores agradecem ao Conselho Nacional de Desenvolvimento Científico e Tecnológico (CNPq) pelo suporte financeiro na concretização deste trabalho, por meio de bolsas e auxílio financeiro. Agradecemos também ao Programa Institucional de Bolsas de Iniciação Científica (PIBIC) pela bolsa concedida a uma das co-autoras. Aos relatores da RBG agradecemos pelas sugestões ao manuscrito. 


\section{Referências}

Allen P.A. \& Hoffman P.F. 2005. Extreme winds and waves in the aftermath of Neoproterozoic glaciation. Nature, 433:123-127.

Alvarenga C.J.S.de, Santos R.V. \& Dantas E.L. 2004. C-O-Sr isotopic stratigraphy of cap carbonates overlying Marinoan-age glacial diamictites in the Paraguay Belt, Brazil. Precambrian Res., 131:1-21.

Alvarenga C.J.S. de, Dardenne M.A., Santos R.V., Brod E.R., Gioia S.M.C.L., Sial A.N., Dantas E.L. \& Ferreira V.P. 2007. Isotope stratigraphy of Neoproterozoic cap carbonate in the Araras Group, Brazil. Gondwana Research (no prelo).

Babinski M, Vieira L.C., Trindade R.I.F. 2007. Direct dating of the sete Lagoas cap carbonate (Bambuí Group, razil) and implications for the Neoproterozoic glacial events. Terra Nova, 19:1-6.

Bartley J.K., Semikhatov M.A., Kaufman A.J., Knoll A.H., Pope M.C. \& Jacobsen S.B. 2001. Global events across the Mesoproterozoic-Neoproterozoic boundary: $\mathrm{C}$ and $\mathrm{Sr}$ isotope evidence from Siberia. Precambrian Res., 111:165-202.

Branco J.J.R. \& Costa da M.T. 1961. Roteiro da Excursão Belo Horizonte-Brasília. In: UFMG, Congresso Brasileiro de Geologia, 14, Belo Horizonte, Inst. Pesq. Radioat, p. 9-25.

Buick R., DesMarais D.J. \& Knoll A.H. 1995. Stable isotopic compositions of carbonates from the Mesoproterozoic Bangemall Group, northwestern Australia. Chemical Geology, 123:153-171.

Cukrov N., Alvarenga C.J.S. de \& Uhlein A. 2005. Litofácies da glaciação neoproterozóica nas porções sul do Cráton do São Francisco: exemplos de Jequitaí, MG e Cristalina, GO. Rev. Bras. Geoc., 35:69-76.

Castro P.T.A. 1997. Os conglomerados da borda SW do Cráton do São Francisco junto à porção S da Faixa Brasília: sedimentologia e relações estratigráficas com as rochas do Grupo Bambuí. Tese de Doutoramento, Instituto de Geociências, Universidade de Brasília, 264 p.

Castro P.T.A. \& Dardenne M.A. 2000. The sedimentology, stratigraphy and tectonic context of the São Francisco Supergroup at the southern boundary of the São Francisco Craton, Brazil. Rev. Bras. Geoc., 30:439-441.

Dardenne M.A. 1978. Síntese sobre a estratigrafia do Grupo Bambuí no Brasil Central. In: SBG, Congr. Bras. Geol., 30, Anais, v. 2, p. 97-610.

Dardenne M.A. 1981. Os grupos Paranoá e Bambuí na faixa dobrada Brasília. In: SBG, Simp. craton São Francisco, 1, Anais, p.104-157.

Dardenne M.A. 2000. The Brasília Fold Belt. In: U.G. Cordani, E.J. Milani, A. Thomaz Filho \& D.A. Campos (eds.) Tectonic Evolution of South America. Rio de Janeiro, Brazil, p. 231-264.

Fairchild I.J., Spiro, B., Herrington P.M. \& Song T. 2000. Controls on $\mathrm{Sr}$ and $\mathrm{C}$ isotope compositions of Neoproterozoic Sr-rich limestones fo east Greenland and north China. In: J.P. Grotzinger \& N.P. James (eds.) Carbonate sedimentation and diagenesis in the evolving Precambrian world. SEPM, Special Publication 67, Tulsa, Okla., p. 297-314.

Fairchild T.R., Schopf J.W., Shen-Miller J., Guimarães E.M., Edwards M.D., Lagstein A., Li X. Pabst M. \& Melo-Filho L.S. 1996. Recent Discoveries of Proterozoic microfossils in south-central Brazil. Precambrian Res., 80:125-152.

Faria A. \& Dardenne M.A. 1995. Estratigrafia do Grupo Paranoá na região de Alto Paraíso de Goiás-São João D’Aliança - GO. In: SBG, Simp. Geol. Centro-Oeste, 5, Anais, p. 75-77.

Gorjan P., Walter M.R. \& Swart R. 2003. Global Neoproterozoic (Sturtian) pos-glacial sulfide-sulfur isotope anomaly recognised in Namibia. J. African. Earth Sci., 36:89-98.

Gorokhov I.M., Semikhatov M.A., Baskakov A.V., Kutyavin E.P., Mel'nikov N.N., Sochava, S.V. \& Turchenko T.L. 1995. Sr isotopic composition in Riphen, Vendian and Lower Cambrian carbonates from Siberia. Strat. Geol, Corr., 3:1-28.

Guimarães E.M. 1997. Estudos de proveniência e diagênese, com ênfase na caracterização dos filossilicatos dos grupos Paranoá e Bambuí, na região de Bezerras-Cabeceiras, GO. Tese de doutoramento, Universidade de Brasília, 260 p.

Guimarães E.M. \& Dardenne M.A. 2004. Tectonic settings indicated by phyllosilicates in Paranoá and Bambuí Group. In: International Geological Congress, 32, $A b$ stracts (CD-ROM).

Guimarães E.M. \& Dardenne M.A. 2005. Glauconitas do Grupo Paranoá: condições de deposição e contexto tectônico. In: SBG, Simpósio sobre o Cráton do São Francisco, 3, Anais, p. 223-226.

Guimarães E.M., Dardenne M.A., Faria A. de, Coelho C.E.S. \& Piaulino P.O.V. 1986. Relações dos grupos Paranoá, Jequitaí e Bambuí na região de Bezerra (GO). In: Congr. Bras.Geol., 34, Anais., p. 853-860.

Halverson G.P., Hoffman P.F., Schrag D.P., Maloof A.C. \& Rice A.H. 2005. Toward a Neoproterozoic composite carbon-isotope record. Geol. Soc. Am. Bull., 117:11811207.

Hill A.C., \& Walter M.R. 2000. Mid-Neoproterozoic ( 830$750 \mathrm{Ma}$ ) isotope stratigraphy of Australia and global correlation. Precambrian Res., 100:181-211.

Hoffman P.F., Kaufman A.J., Halverson G.P. \& Scrag D.P. 1998. A Neoproterozoic snowball Earth. Science, 281:1342-1346.

Hoffman P.F., Halverson G.P. \& Grotzinger J.P. 2002 . Are Proterozoic cap carbonates and isotopic excursions a record of gas hydrate destabilization following Earth's coldest intervals? Comment and reply. Geology, 30:286-288.

Hoffman P.F. \& Schrag D. P. 2002. The Snowball Earth hypothesis: testing the limits of global change. Terra Nova, 14:129-155.

Isotta C.A.L., Rocha-Campos A.C. \& Yoshida R. 1969. Striated pavement of the Upper Precambrian glaciations in Brazil. Nature, 222:467-468.

Iyer S.S., Babinski M., Krouse H.R. \& Chemale F. 1995. Highly 13C-enriched carbonate and orgaanic matter in the Neorproterozoic sediments of the Bambuí Group, Brazil. Precambrian Res., 73:271-282. 
Jacobsen S.B. \& Kaufman A. 1999. The Sr, C and O isotopic evolution of Neoproterozoic seawater. Chem. Geol., 161:37-57.

James N.P., Narbonne G.M. \& Kyser T.K. 2001. Late Neoproterozoic cap carbonates: Mackenzie Mountains, northwestern Canada: precipitation and global meltdown. Can. J. Earth Sci., 38:1229-1262.

Kah L.C., Sherman A.B., Narbonne G.M., Knoll A. \& Kufman A.J. 1999. $\delta^{13} \mathrm{C}$ stratigraphy of the Proterozoic Bylot Supergroup, Baffin Island, Canada: implications for regional lithostratigraphic correlations. Can. J. Earth Sci., 36:313-332.

Karfunkel J., Hoppe A. \& Noce C.M. 2003. Serra da Água Fria e vizinhanças, MG - Vestígios da glaciação neoproterozóica. In: C. Schobbenhaus, D.A. Campos, E.T. Queiroz, M. Winge \& M.L.C. Berbert-Born (eds.) Sítios Geológicos e Paleontológicos do Brasil. MME-DNPMCPRM-SIGEP, p. 165-174.

Kaufman J.A. \& Knoll H. A. 1995. Neoproterozoic variations in the C-isotopic composition of seawater: straitigraphic and biogeochemical implications. Precambrian Res., 73:27-49.

Knoll A.H., Hayes J.M., Kaufman A.J., Swett K. \& Lambert I.B. 1986. Secular variation in carbon isotope ratios from upper Proterozoic successions of Svalbard and East Greenland. Nature, 321:832-837.

Margaritz, M. Holser W.T. \& Kirschvink J.L. 1986. Carbonisotope events across the Precambrian/Cambrian boundary on the Siberian Platform. Nature, 320:258-259.

Martins Neto M.A. \& Alkmim F.F. 2001. Estratigrafia e evolução tectônica das bacias neoproterozóicas do paleocontinente São Francisco e suas margens: Registro da quebra de Rodínia e colagem de Gondwana. In: C.P. Pinto \& M.A. Martins-Neto (eds.) Bacia do São Francisco: Geologia e Recursos Naturais. SBG-MG, p. 31-54.

McKirdy D.M., Burgess J.M., Lemon N.M., Yu X., Cooper A.M., Gostin V.A., Jenkins R.J.F. \& Both R.A. 2001. A chemostratigraphic overview of the late Cryogenian interglacial sequence in the Adelaide Fold-Thrust Belt, South Australia. Precambrian Res., 106:149-186.

Misi A. 2001. Estratigrafia Isotópica das Seqüências do Supergrupo São Francisco, coberturas neoproterozóicas do Cráton do São Francisco. Idade e Correlações. In: C.P. Pinto \& M.A. Martins-Neto (eds.) Bacia do São Francisco: Geologia e Recursos Naturais. SBG/MG, p. 67-92.

Misi A. \& Veizer J. 1998. Neoproterozoic carbonate sequences of the Una Group, Irecê Basin, Brazil: chemostratigraphy, age and correlations. Precambrian Res., 89:87-100.

Nogueira A.C.R., Riccomini C., Sial A.N., Moura C.A.V. \& Fairchild T.R. 2003. Soft-sediment deformation at the base of the Neoproterozoic Puga cap carbonate (southwestern Amazon craton, Brazil): confirmation of rapid icehouse to greenhouse transition in snowball Earth. Geology, 31:613-616.

Pimentel M.M., Heaman L., Fuck R.A. \& Marini O.J. 1991. $\mathrm{U}-\mathrm{Pb}$ zircon geochronology of Precambrian tin-bearing continental-type acid magmatism in Central Brazil. Precambrian Res., 52:321-335.

Santos R.V., Alvarenga C.J.S. de, Babinski M., Ramos M.L.S., Cukrov N., Fonseca M.A., Sial A.N., Dardenne M.A. \&
Noce C.M. 2004. Carbon isotope of MesoproterozoicNeoproterozoic sequences from Southern São Francisco craton and Araçuaí Belt, Brazil: Paleographic implications. J. South Am. Earth Sci., 18:27-39.

Santos R.V., Alvarenga C.J.S. de, Dardenne M.A., Sial A.N. \& Ferreira V.P. 2000. Carbon and oxygen isotope profiles across Meso-Neoproterozoic limestones from central Brazil: Bambuí and Paranoá Groups. Precambrian Res., 104:107-122.

Souza J.D., Kosin M., Heineck C.A., Lacerda Filho J.V., Teixeira L.R., Valente C.R., Guimarães J.T. Bento R.V., Borges V.P., Santos R.A., Leite C.A., Neves L.P., Oliveira I.W.B., Carvalho L.M., Pereira L.H.M. \& Paes V.J.C. 2004. Folha SD. 23 - Brasília In: C. Schobbenhaus, J.H. Gonçalves, J.O.S. Santos, M.B. Abram, R. Leão Neto, G.M.M. Matos, R.M. Vidoti, M.A.B. Ramos \& J.D.A. de Jesus (eds.) Carta Geológica do Brasil ao Milionésimo, SIG, Programa Geologia do Brasil, CD-ROM.

Thomas C.W., Graham C.M., Ellam R.M. \& Fallick A.E. 2004. ${ }^{87} \mathrm{Sr} /{ }^{86} \mathrm{Sr}$ chemostratigraphy of Neoproterozoic Dalradian limestones of Scotland and Ireland: constrains on depositional age and time scales. J. Geol. Soc. London, 161:229-242.

Uhlein A., Trompette R. \& Alvarenga C.J.S. 1999. Neoproterozoic glacial and gravitational sedimentation on a continental rifted margin: the Jequitaí-Macaúbas sequence (Minas Gerais, Brazil). J. South Am. Earth Sci., 12:435451.

Uhlein A., Alvarenga C.J.S. de, Trompette R., Dupont H.S.J.B., Egydio-Silva M., Cukrov N. \& Lima O.N.B.L. 2004. Glaciação neoproterozóica sobre o Cráton do São Francisco e faixas dobradas adjacentes. In: V. MantessoNeto, A. Bartorelli, C.D.R. Carneiro \& B.B. Brito-Neves (orgs.). Geologia do Continente Sul-Americano: Evolução da obra de Fernando Flávio Marques de Almeida. BECA, p. 539-553.

Valeriano C.M., Dardenne M.A., Fonseca M.A., Simões L.S.A. \& Seer H.J. 2004. A Evolução Tectônica da Faixa Brasília. In: V. Mantesso-Neto, A. Bartorelli, C.D.R. Carneiro \& B.B. Brito-Neves (orgs.). Geologia do Continente Sul-Americano: Evolução da obra de Fernando Flávio Marques de Almeida. BECA, p. 575-592.

Veizer J., Holser W.T. \& Wilgus C.K. 1980. Correlation of ${ }^{13} \mathrm{C} /{ }^{12} \mathrm{C}$ and ${ }^{34} \mathrm{~S} /{ }^{32} \mathrm{~S}$ secular variations. Geoch. Cosmoch. Acta., 44:579-587.

Walter M.R., Veevers J.J., Calver C.R., Gorjan P. \& Hill A.C. 2000. Dating the 840-544 Ma. Neoproterozoic interval by isotopes of strontium, carbon, and sulfur in seawater, and some interpretative models. Precambrian Res., 100:371-433.

Yoshioka H., Asahara Y., Tojo B. \& Kawakami S. 2003. Systematic variations in $\mathrm{C}, \mathrm{O}$, and $\mathrm{Sr}$ isotopes and elemental concentrations in Neoproterozoic carbonates in Namibia: implications for a glacial to interglacial transition. Precambrian Res., 124:69-85.

Manuscrito CSF-19

Submetido em 31 de maio de 2006 Aceito em 09 de abril de 2007 